\title{
(Cost)-effectiveness of self-treatment of exacerbations on the severity of exacerbations in patients with COPD: the COPE II study
}

\author{
T Effing, ${ }^{1,2} \mathrm{H}$ Kerstjens, ${ }^{3} \mathrm{P}$ van der Valk, ${ }^{1} \mathrm{G}$ Zielhuis, ${ }^{4} \mathrm{~J}$ van der Palen ${ }^{1,5}$
}

${ }^{1}$ Medisch Spectrum Twente, Department of Pulmonology, Enschede, The Netherlands;

${ }^{2}$ Repatriation General Hospital, Department of Respiratory Medicine, Daw Park, South Australia, Australia; ${ }^{3}$ University Medical Center Groningen and University of Groningen, Department of Pulmonology, Groningen, The Netherlands;

${ }^{4}$ Radboud University Nijmegen, Department of Epidemiology, Biostatistics, and HTA

Nijmegen, The Netherlands; ${ }^{5}$ Department of Research Methodology, Measurement and Data Analysis, University of

Twente, Enschede, The

Netherlands

\section{Correspondence to:}

Dr T Effing, Repatriation General

Hospital, Department of

Respiratory Medicine, Daws

Road, Daw Park, SA 5041

Australia; tanja.effing@

health.sa.gov.au

Received 15 December 2008

Accepted 9 August 2009

Published Online First

6 September 2009
ABSTRACT

Background: Chronic obstructive pulmonary disease (COPD) is a chronic disease with a high prevalence and rapidly increasing incidence rates. The effect of selftreatment of COPD exacerbations on the severity of exacerbations during a 1-year period was examined and a cost-effectiveness analysis was performed.

Methods: Patients were randomly allocated to four 2hour self-management sessions, with or without training in self-treatment of exacerbations. Patients in the selftreatment group received an action plan with the possibility to initiate a course of prednisolone (with or without antibiotics). During follow-up, all participants kept a daily symptom diary. These provided the data to calculate the frequency of exacerbations, the number of exacerbation days and mean daily severity scores.

Results: Data were analysed for 142 randomised patients (self-treatment: $n=70$; control: $n=72$ ). The frequency of exacerbations was identical in both study groups (mean (SD) 3.5 (2.7)). Patients in the self-treatment group reported fewer exacerbation days (median 31 (interquartile range (IQR) 8.9-67.5) in the self-treatment group vs 40 (IOR 13.3-88.2) in the control group; $p=0.064$ ); the difference was significant in the group of patients with a high number of exacerbation days per year (>137 (90th percentile of the whole study population); $p=0.028$ ). The mean severity score of an exacerbation day was equal in both groups. No between-group differences were found in health-related quality of life. Cost-effectiveness analyses showed that applying self-treatment saved $€ 154$ per patient, with a trend towards a lower probability for hospital admissions (0.20/patient/year in the self-treatment group vs 0.33/patient/year in the control group; $p=0.388)$ and a significant reduction of health care contacts (5.37/patient/year in the self-treatment group vs $6.51 /$ patient/year in the control group; $p=0.043$ ).

Conclusion: Self-treatment of exacerbations incorporated in a self-management programme leads to fewer exacerbation days and lower costs.

The course of chronic obstructive pulmonary disease (COPD) is characterised by episodes of acute deterioration in respiratory health termed "exacerbations". These exacerbations account for much of the morbidity, mortality and hospital admissions in patients with COPD. ${ }^{1}$ Most of the costs in patients with COPD are related to the treatment of acute exacerbations, with the costs of hospitalisations being the major component. ${ }^{2}$

Patients seem to be capable of identifying warning signs and symptoms of exacerbations, ${ }^{3}$ but they often do not report an exacerbation to their physician and are therefore potentially delaying treatment. ${ }^{14}$ Proper guidelines for self-treatment of exacerbations might help to induce prompt treatment of exacerbations, thereby accelerating recovery and probably reducing the risk of hospitalisation. Self-treatment of exacerbations therefore has the potential to reduce their severity and associated costs.

To date there is no consensus about the exact definition of a COPD exacerbation or its severity. Several frequently quoted definitions of exacerbations are based on respiratory symptoms, ${ }^{15}$ and therefore the number and length of exacerbations can be defined by using daily symptom scores. ${ }^{1}$ Severity of exacerbations is often characterised by type of health care consumption (eg, courses of steroids, hospitalisations), ${ }^{6}$ but the number of exacerbation days in combination with daily symptom scores as a measure of severity might be a more precise alternative. Because the presence of symptoms is more the rule than the exception in most patients with COPD, using the change in level of symptoms relative to baseline instead of the absolute level could increase the accuracy of the outcome measurement further. ${ }^{4}$

We evaluated the effects of self-treatment of exacerbations within a self-management programme in a randomised study, the COPD study at the department of Pulmonology in Enschede (COPE) II study. The severity of exacerbations (total number of exacerbation days combined with the average daily severity score) was assessed by determining change relative to baseline in daily symptoms during a 1-year follow-up. In addition, a cost-effectiveness analysis was performed.

\section{METHODS \\ Patients}

From November 2004 through July 2006, 159 patients were recruited from the outpatient department of pulmonary medicine of Medisch Spectrum Twente Hospital, Enschede, The Netherlands. Patients had to meet the following criteria: (1) a clinical diagnosis of COPD according to the GOLD criteria; ${ }^{6}(2)$ no exacerbation in the month before enrolment; (3) $>3$ exacerbations, defined as respiratory problems that required a course of oral corticosteroids and/or antibiotics, or $>1$ hospitalisation for respiratory problems in the 2 years preceding study entry; (4) (ex) smoker; (5) age 40-75 years; (6) post-bronchodilator forced expiratory volume in $1 \mathrm{~s}\left(\mathrm{FEV}_{1}\right) 25-80 \%$ predicted; (7) able to understand and read Dutch; and (8) written informed consent from the subject prior to participation. Patients were excluded if 
they had: (1) serious other disease with a low survival rate; (2) other diseases influencing bronchial symptoms and/or lung function (eg, cardiac insufficiency, sarcoidosis); (3) severe psychiatric illness; (4) uncontrolled diabetes mellitus during a COPD exacerbation in the past or a hospitalisation for diabetes mellitus in the 2 years preceding the study; (4) need for regular oxygen therapy ( $>16 \mathrm{~h} /$ day or oxygen tension $<7.2 \mathrm{kPa}$ ); (5) maintenance therapy with antibiotics; (6) known $\alpha_{1^{-}}$ antitrypsin deficiency; (7) disorders or progressive disease seriously influencing walking ability (eg, amputation, paralysis, progressive muscle disease).

\section{Study design}

A $2 \times 2$ factorial design was used, meaning that two independent interventions (self-treatment and a community-based physiotherapeutic exercise programme (COPE-active)) were evaluated using one design (table 1). The effect of a self-management programme including self-treatment of exacerbations (selftreatment group) was compared with a self-management programme only (control group). Patients receiving COPEactive were divided equally over the self-treatment and control group using a minimisation programme, ${ }^{7}$ minimising differences between groups in gender, current smoking, $\mathrm{FEV}_{1}$ predicted ( $\leqslant$ or $>50 \%$ ) and use of inhaled corticosteroids. This design assumes that both interventions do not interact with each other, which needed to be verified before the final analyses. Measurements of health-related quality of life, anxiety and depression, and lung function were performed at baseline and after 7 and 12 months.

\section{Self-management sessions and self-treatment intervention}

Smokers were offered a 3-month smoking cessation programme prior to group allocation. ${ }^{8}$ After randomisation, patients in both study groups and their partners were offered four weekly 2-hour small group (approximately five patients) self-management sessions given by a respiratory nurse and a physiotherapist. The intention of the courses was to change the patients' disease behaviour by increasing their knowledge, confronting them with consequences of specific behaviour and supplying them with tools to deal with different components of their disease. Patients in the intervention group additionally received training in self-treatment of exacerbations. The respiratory nurse contacted all patients by telephone 4, 13 and 26 weeks after the last course to recall the items addressed during the selfmanagement courses. Patients were supplied with a booklet with the content of the courses.

All patients were educated in completing daily diaries in which they had to report whether their major symptoms (breathlessness, sputum production, sputum colour) and minor symptoms (cough, wheeze, running nose, sore throat and fever $\left(>38.5^{\circ} \mathrm{C}\right)$ ) according to Anthonisen et al were above normal. At inclusion, all patients received a "what is normal" card which described their individual levels of major symptoms in a stable healthy state. When patients experienced no deterioration in any of the symptoms listed in the diary they could tick "no change in symptoms", otherwise they had to report on all the symptoms in the diary whether the level of each symptom was normal, slightly increased or clearly increased.

The start of the exacerbation was defined as "a clear negative change in two major symptoms or one major and one minor symptom from baseline for at least two consecutive days". The day that the exacerbation was resolved was defined as the first day of (1) three successive days that the patient had returned to his normal health state or (2) seven consecutive days on which patients continuously reported no or only a slight increase in symptoms compared with baseline with no fever or change in sputum colour.

Patients in the self-treatment group were educated in early recognition of exacerbations and were taught when to start a course of oral prednisolone by using an action plan that was linked to the daily diary. For treatment of an exacerbation diagnosed either by the physician or by the patient from the action plan, a course of prednisolone (30 mg for 7 days) was the standard medication. Change in sputum colour was the indicator to combine prednisolone with antibiotics (amoxicillin/clavulanic acid 500/125 mg (Augmentin) three times daily for 7 days or, in cases of hypersensitivity, doxycycline $100 \mathrm{mg}$ daily for 10 days).

Patients in the control group were instructed to call the study office if they felt in need of assistance for any worsening of their respiratory symptoms, which normally would have prompted them to call their chest physician or general practitioner instead. A consultation with a chest physician was scheduled within $12 \mathrm{~h}$.

\section{Outcomes}

The total number of exacerbation days was calculated by summing up all exacerbation days per patient per year, based on the information from the diaries. The severity of an exacerbation day was calculated with the help of symptom scores. The major symptoms were scored as: normal $=0$; small increase $=1$; or clear increase $=2$. The minor symptoms were scored 0, 0.5 and 1, respectively. Sputum colour was scored as: normal $=0$, different from normal $=2$; and fever: no $=0$, yes $=1$. Adding all these scores resulted in a daily score with a range of $0-11$ points. When patients were admitted to the hospital, a daily score of 15 points was assigned. If patients had less than 4 months of complete diary data they were excluded from the analyses.

Table 1 Design of the COPE II study, including the two interventions (self-treatment of exacerbations and COPE-active) and the measurements at baseline, 7 and 12 months

\begin{tabular}{|c|c|c|c|c|c|}
\hline \multirow[b]{2}{*}{ Time schedule } & \multirow[b]{2}{*}{ Interventions } & \multicolumn{2}{|c|}{$\begin{array}{l}\text { Self-treatment group } \\
\text { (intervention group) }\end{array}$} & \multicolumn{2}{|c|}{ Control group } \\
\hline & & Subgroup 1 & Subgroup 2 & Subgroup 3 & Subgroup 4 \\
\hline Month 0 & Baseline measurement & $x$ & $\times$ & $x$ & $\times$ \\
\hline Month 1 & Self-management courses & $x$ & $\times$ & $\times$ & $\times$ \\
\hline Month 1-12 & Self-treatment of exacerbations & $\times$ & $\times$ & - & - \\
\hline Month 2-7 & COPE-active: "compulsory" & $\times$ & - & $x$ & - \\
\hline Month 7 & Measurement & $x$ & $\times$ & $\times$ & $\times$ \\
\hline Month 8-12 & COPE-active: "voluntary" & $x$ & - & $x$ & - \\
\hline Month 12 & Measurement & $x$ & $\times$ & $\times$ & $\times$ \\
\hline
\end{tabular}


Health-related quality of life (HRQoL) was measured with the self-administered Chronic Respiratory Questionnaire standardised (CRO-SAS). ${ }^{9}$ The questionnaire captures four domains: dyspnoea, fatigue, emotional functioning and mastery. A change of 0.5 in a domain is considered to be clinically relevant at the individual level. ${ }^{10}$ Health status was evaluated by the selfadministrated Clinical COPD Questionnaire (CCQ). ${ }^{11}$ A change of 0.4 represents the minimal important difference for an individual patient. ${ }^{12}$ Anxiety and depression was measured with the Hospital Anxiety and Depression Scale (HADS). ${ }^{13}$ This instrument produces separate scores for anxiety and depression ranging from 0 to 21 . A score of $>10$ is judged to be a predictor of a clinical diagnosis of anxiety and depression. Finally, information about hospital admissions, emergency room visits, scheduled and emergency outpatients visits, telephone calls with physicians, consultations with the general practitioner and information about medication use was collected for costeffectiveness analyses.

\section{Statistical analyses}

We calculated that 79 patients per treatment group were required to detect a difference of 2 exacerbation days (SD 4.5 days) with $80 \%$ power and a two-sided $0.05 \alpha$-level test. All statistical analyses were performed on an intention-to treat basis using SPSS Version 12.0. Between-group differences of "exacerbation days/year" and "mean severity score/year" were assessed by comparing the proportion of patients with an individual value larger than the median, the 75th and the 90th percentile. Subsequently, $\chi^{2}$ statistics for categorical variables were applied. Between-group differences of continuous variables were assessed by analysis of repeated measurements using the SPSS mixed models procedure and, in case of continuous variables (number of hospitalisations, courses of prednisolone/ antibiotics), by the Student $t$ test (normal distribution) or the Wilcoxon rank sum test (non-normal distribution). In cases of non-normal distribution, the median combined with the interquartile range (IOR) was presented.

\section{Cost-effectiveness}

A decision analytical model with a time perspective of 12 months was developed to evaluate the cost-effectiveness of a self-management programme including self-treatment of exacerbations versus a self-management programme only. Resource use was multiplied by 2004 unit prices. ${ }^{14}$ Medication costs were based on market prices and included $\mathrm{a} € 6$ dispensing fee and $6 \%$ VAT for each prescription. We took the perspective of the health care payer. The costeffectiveness ratio was calculated as costs per exacerbation day prevented, per hospital admission prevented and per health care contact prevented.

For sensitivity analysis, a Monte Carlo simulation with 1000 iterations was performed to evaluate the relative impact of likely variations in the parameters in the decision analytical model. Cost parameters and probabilities were therefore varied simultaneously over their ranges and associated $95 \%$ confidence intervals (CI). For the costs of health care contacts and hospital admissions a normal distribution was used while, for the costs of the self-management programme, exacerbations and regular medication a triangular distribution was used. Logistic normal distributions were used for all probabilities.

\section{RESULTS}

Of the 421 eligible patients, 80 were assigned to the selftreatment group and 79 to the control group (fig 1). Between inclusion and the baseline measurements, three patients dropped out in each group and insufficient diary data were delivered by 11 patients. The baseline characteristics of the remaining 142 patients are shown in table 2, and were similar in both groups with respect to all measured prognostic factors. The 11 dropouts did not differ from the remaining group in any factors except the Medical Research Council dyspnoea scale (mean (SD) 2.3 (1.1) vs 3.0 (1.1) in dropouts versus remaining patients; $p=0.041)$. During the 1 -year follow-up period, three patients in the self-treatment group dropped out, of whom one died of an intracerebral haemorrhage. Thus, 67 patients in the self-treatment group and 72 patients in the control group completed the 1-year follow-up.

The mean (SD) frequency of exacerbations was the same in both study groups $(3.5(2.7))$. Figure 2 shows that patients in the self-treatment group had a lower total number of exacerbation days than patients in the control group. Differences were significant in the group of patients with
Figure 1 Flow diagram of progress of subjects through the COPE II study.

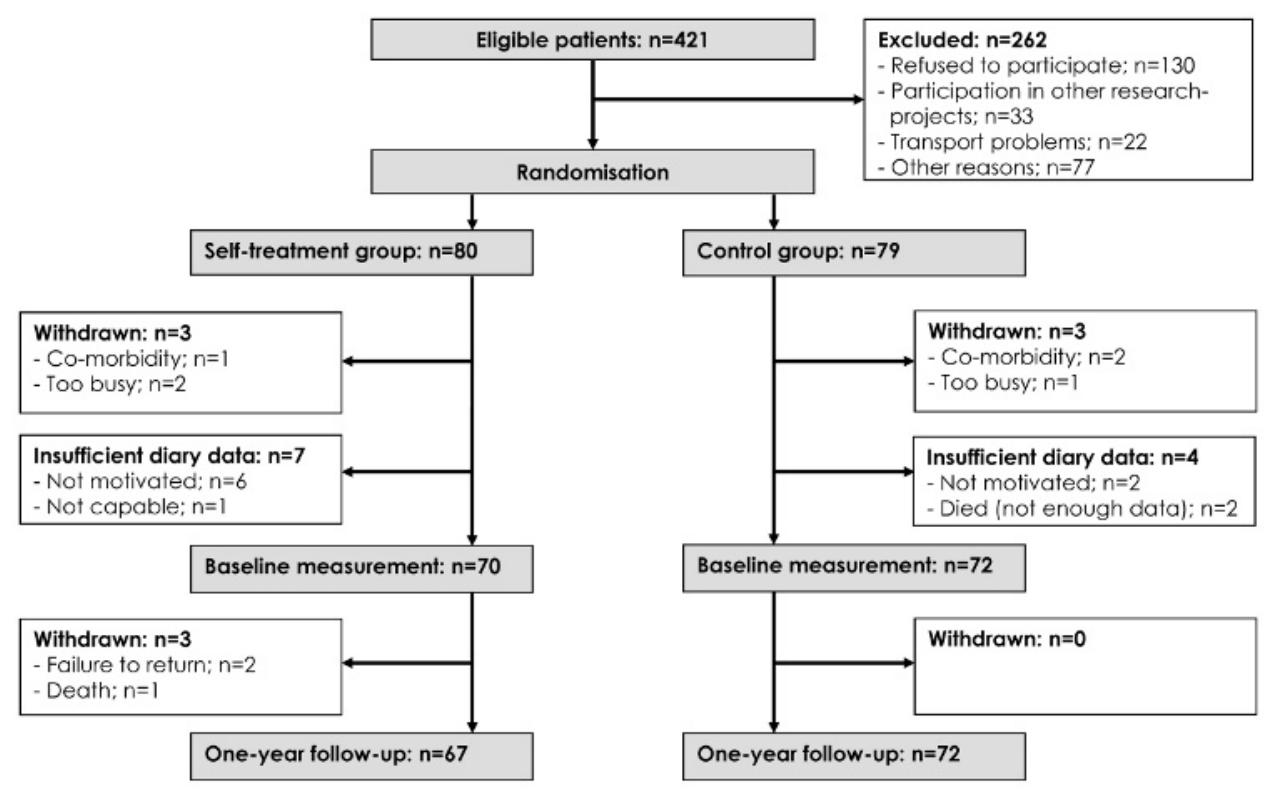


Table 2 Baseline characteristics of the two treatment groups

\begin{tabular}{lcc}
\hline & $\begin{array}{l}\text { Self-treatment } \\
(\mathbf{n}=\mathbf{7 0})\end{array}$ & $\begin{array}{l}\text { Control } \\
(\mathbf{n}=\mathbf{7 2})\end{array}$ \\
\hline Mean (SD) age (years) & $63.1(7.9)$ & $63.7(8.0)$ \\
Male (\%) & 57.1 & 61.1 \\
Smokers (\%) & 32.9 & 33.3 \\
Mean (SD) MRC dyspnoea scale & $2.31(1.06)$ & $2.33(1.14)$ \\
Mean (SD) lung function post & & \\
bronchodilation & & \\
$\quad$ FEV ${ }_{1}($ I) & $1.44(0.56)$ & $1.41(0.50)$ \\
FEV 1 (\% predicted) & $50.7(16.3)$ & $49.6(15.3)$ \\
$\quad$ VC (I) & $3.64(1.08)$ & $3.66(0.85)$ \\
Inhaled corticosteroid use (\%) & 88.6 & 84.7 \\
\hline
\end{tabular}

FEV $_{1}$, forced expiratory volume in $1 \mathrm{~s}$; MRC, Medical Research Council; VC, vital capacity.

a relatively high number of exacerbation days per year (>137 days/year (P90 of the whole study population); $p=0.028)$. The mean (SD) severity score of an exacerbation day did not differ between the two study groups (self-treatment 5.1 (2.4) vs control 5.1 (3.0)). Additional analyses of the more severe exacerbation days (daily score $>6$ points, which represents a maximum score on all major symptoms) showed a (non-significant) trend towards a higher median number of severe exacerbation days/year in the control group (selftreatment 11 (IOR 0.8-21.0) vs control 15 (IOR 3.3-26.8); $\mathrm{p}=0.124)$

The self-reported median number of prednisolone courses (selftreatment 2.6 (IOR 1.0-5.0) vs control 1.7 (IOR 1.0-3.2); p = 0.055) and antibiotic courses (self-treatment 2.0 (IOR 0.8-4.0) vs control 1.1 (IOR 0.0-2.9); $p=0.048$ ) were higher in the self-treatment group. The number of hospital admissions was 14 in the selftreatment group and 24 in the control group $(p=0.388)$. A significant reduction of health care contacts was detected (5.37/ patient/year in the self-treatment group vs 6.51/patient/year in the control group; $p=0.043$ ). No between-group differences were

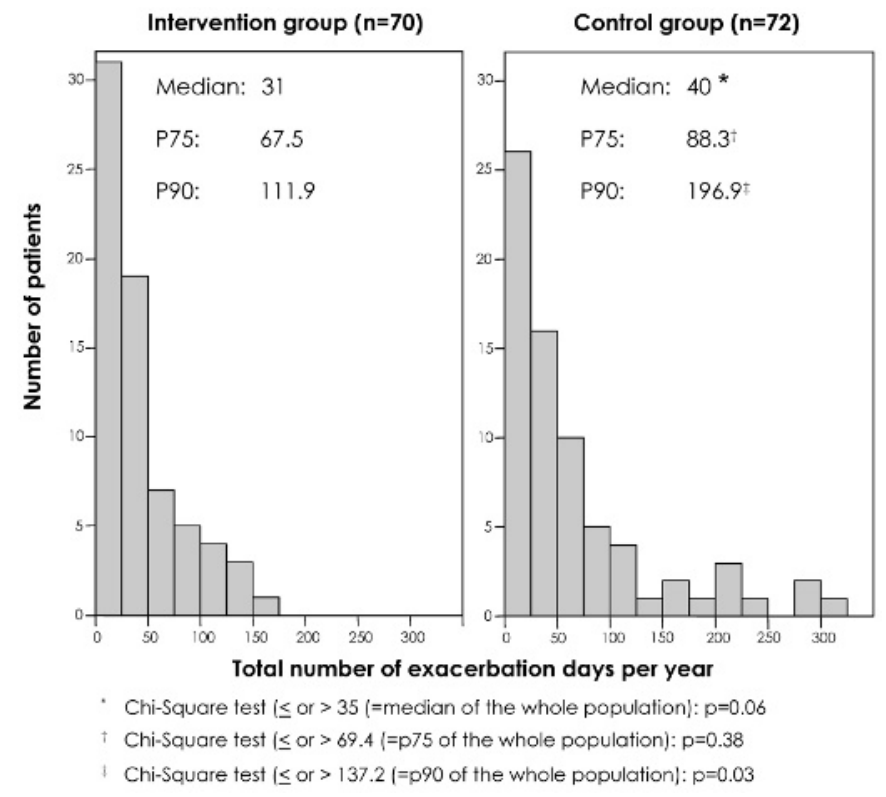

Figure 2 Number of exacerbation days per year for patients in the intervention and control groups; median, 75th (P75) and 90th percentile (P90) of the two study groups and the corresponding $p$ values for group differences are shown. found in the CRQ components, CCQ (sub)scores, HAD scores and $\mathrm{FEV}_{1}$ percentage predicted over the 12 -month period.

\section{Cost-effectiveness}

Table 3 shows the percentages derived from the COPE II study for each step in the decision analytical model (fig 3). Figure 3 also shows the percentage of patients in the different branches. For example, the percentage of patients in the self-treatment group having at least one exacerbation $(90 \%)$, one health care contact $(100 \%)$ and one hospital admission $(17.7 \%)$ is $15.9 \%$ $(90 \% \times 100 \% \times 17.7 \%)$. The cost incurred by a patient in this branch is $€ 6388$, consisting of: the cost of a self-management course including self-treatment (€118), regular medication cost (€761), cost of health care contacts (€281) (table 4) and cost of hospitalisation (€5227). In this branch, medication for an exacerbation is incorporated in hospitalisation costs. The percentage of patients in each branch will finally determine the weight of the branch costs to the total cost/patient/study group (eg, $15.9 \% \times € 6388$ ).

The 12 -month cost data are presented in table 4 and the effect data in table 5. The self-treatment strategy was dominant over the control strategy. Patients in the self-treatment group generated $€ 2040$ in direct medical costs compared with $€ 2194$ in the control group. Thus, the use of self-treatment saved $€ 154$ per patient. The number needed to treat (NNT) to prevent one hospitalisation was 7.4, and the NNT to prevent one health care contact was 0.9 . The results of the sensitivity analysis with regard to costs for hospital admissions and health care contacts are shown in fig 4 . The main outcome was that a positive treatment effect in combination with saving money was seen in most of the outcomes (hospital admissions 63.3\%; health care contacts $49.2 \%$ ). Positive effects, independent of costs, were seen in $95.1 \%$ and $74.4 \%$ of the iterations for hospital admissions and health care contacts, respectively.

\section{DISCUSSION}

This study shows that self-treatment of exacerbations leads to fewer exacerbation days in combination with an increase in the use of courses of prednisolone and antibiotics. No betweengroup differences in the number of exacerbations, mean daily severity score and HRQoL were found. Cost-effectiveness analyses showed that the self-treatment strategy was dominant over (ie, compared favourably with) the control strategy, with lower probabilities for hospital admissions and health care contacts in the self-treatment group combined with cost savings.

Before the study we hypothesised that the severity of exacerbations, defined by the length of the exacerbation in combination with the daily severity scores, would be influenced by the self-treatment guidelines because self-treatment would lead to a more rapid start of treatment after the early recognition of an exacerbation. A priori, we did not expect an effect on exacerbation frequency because self-treatment was initiated after the onset of an exacerbation. Both hypotheses were confirmed by our results. Our study data showed a reduction in the number of exacerbation days in the selftreatment group. Remarkably, this was not accompanied by a reduction in the mean daily severity score. A closer look suggests that this lack of difference in daily severity score between the study groups is at least partly explained by the fact that selftreatment particularly reduces the number of the less severe exacerbation days (towards the resolution phase of the exacerbation) in the self-treatment group. As a consequence, 
Table 3 Base case values of percentages of patients with a COPD exacerbation, hospitalisation and medication use for an exacerbation

\begin{tabular}{|c|c|c|}
\hline & \multicolumn{2}{|c|}{ Base case value $(95 \% \mathrm{CI})$} \\
\hline & Self-treatment & Control \\
\hline Patients with an exacerbation (\%) & $90.0(83.1$ to 96.9$)$ & 84.7 (76.3 to 93.1$)$ \\
\hline $\begin{array}{l}\text { Patients with a hospitalisation when } \\
\text { having an exacerbation and health care } \\
\text { contact (\%) }\end{array}$ & 17.7 (8.8 to 26.6 ) & 24.6 (14.7 to 34.5$)$ \\
\hline $\begin{array}{l}\text { Patients with medication use for an } \\
\text { exacerbation when not hospitalised but } \\
\text { having an exacerbation and a health } \\
\text { care contact }(\%)\end{array}$ & 90.8 (84.0 to 97.6$)$ & $61.4(50.2$ to 72.6$)$ \\
\hline
\end{tabular}

the mean daily severity score (total severity score/number of exacerbation days/patient) of the remaining days of the selftreatment group goes up. We hypothesise that the mean severity score in the control group was higher due to the fact that patients in this group experienced both a higher number of more severe exacerbation days as well as a higher number of less severe exacerbation days. This hypothesis is supported by the (non-significant) trend towards a larger number of more severe exacerbation days (symptom score $>6$ points) in the control group.

The difference in severity of exacerbations between the study groups might have been underestimated. By participating in the self-management programme, patients in the control group might have become more aware of the negative consequences of exacerbations and might have contacted the hospital more quickly than normal. Furthermore, because of their participation in this study, control patients experienced a very low threshold for contacting a chest physician and a very short delay in actually being seen $(<12 \mathrm{~h})$ compared with usual care practice. This all might have led to a more rapid start of treatment and, as a consequence, a less severe exacerbation.

All patients including those in the control group received a self-management programme. This increased the costs in the control group also, and raises the question whether a control group without a self-management programme would have constituted a clearer design. However, a comparison between our self-treatment group and a control group receiving no intervention at all would probably have led to similar conclusions with regard to cost-effectiveness, since the favourable effects of the intervention would probably have increased further. Furthermore, the costs of the self-management programme (€112) were very low and avoiding them would therefore have saved little.

Similar to other studies ${ }^{15}$ including COPE I, ${ }^{16}$ our data showed a significantly higher reported use of courses of prednisolone and antibiotics in the self-treatment group. However, since the number of reported courses in the self-treatment group was still lower than the actual number of exacerbations reported in the diaries-meaning that prednisolone was not used during each exacerbation-we are convinced that the between-group difference in courses is due to the underreporting of respiratory

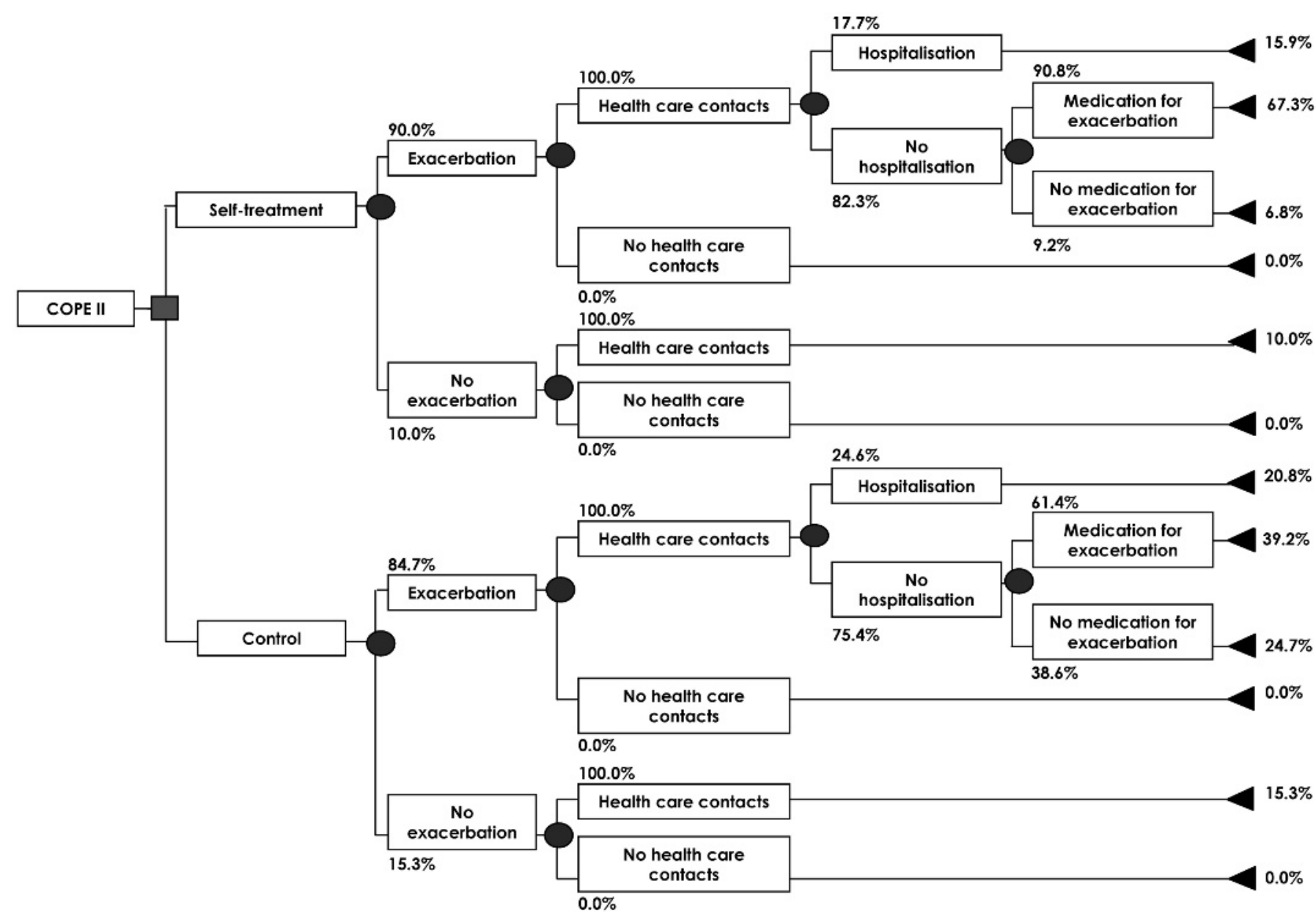

Figure 3 Decision analytical model with percentages of patients with exacerbations, health care contacts, hospitalisations and medication for exacerbations for the self-treatment and control groups. 
Table 4 Mean costs $(€)$ per patient per year in patients with and without self-treatment using 2004 prices

\begin{tabular}{lccr}
\hline & $\begin{array}{l}\text { Self-treatment } \\
(\mathbf{n}=\mathbf{7 0 )}\end{array}$ & $\begin{array}{l}\text { Control } \\
(\mathbf{n}=\mathbf{7 2})\end{array}$ & Difference \\
\hline Self-management courses & 117.68 & 111.60 & 6.08 \\
Regular medication* & 761.21 & 644.00 & 117.21 \\
Health care contacts $\dagger$ & 262.49 & 366.70 & -104.21 \\
$\quad$ Patients with an exacerbation & $280.52(90 \%)$ & $402.94(84.7 \%)$ & \\
$\quad$ Patients without an exacerbation & $100.21(10 \%)$ & $166.07(15.3 \%)$ & \\
Hospitalisation costs $\$$ & 823.24 & 1020.36 & -197.12 \\
Medication for exacerbations§ & 75.30 & 51.40 & 23.90 \\
Total costs $(€)$ & 2039.92 & 2194.06 & -154.14 \\
\hline
\end{tabular}

\footnotetext{
*Includes $€ 6$ pharmacy cost and 6\% VAT per prescription.

flncludes (telephone) consultations with the physician and general practitioner, and emergency room visits.

\#Costs per hospitalisation day: $€ 337$ (includes medication costs); mean of 12.21 and 9.08 hospitalisation days per admission and 14 and 24 admissions for the self-treatment and control groups, respectively. The sum of $€ 5227$ for hospitalisation costs (as mentioned in the example in the text) are the hospitalisation costs per self-treatment patient having at least one hospitalisation: average cost of a hospitalisation $\times$ (total number of hospitalisations in the self-treatment group/total number of intervention patients with $>1$ exacerbation).

$\S$ Mean of medically treated exacerbations per patient per year for the self-treatment and control groups, respectively: treatment with prednisolone and antibiotics: 2.31 vs 1.43 (€27.69 per exacerbation); treatment with prednisolone only: 0.93 vs 0.82 (€7.79 per exacerbation); and treatment with antibiotics only: 0.21 vs 0.27 (€19.90 per exacerbation).
}

complaints to the chest physician by patients in the control group rather than a reflection of overtreatment in the selftreatment group. This underreporting has also been observed in several other studies. ${ }^{13}{ }^{17}$

No effects were found on HRQoL and lung function. Associations between exacerbation frequency and $\mathrm{HROOL}^{1} 1819$ and between severity of exacerbations and $\mathrm{HROOL}^{18}$ have been reported by others. Since we found no effect on exacerbation frequency, a relation with changes in HRQoL or lung function was no longer expected.

In this study the change from baseline was used to define symptoms and therefore exacerbation severity. This method was introduced because almost all patients with COPD always experience at least some symptoms in the stable state. Using health care consumption (courses of prednisolone/antibiotics)
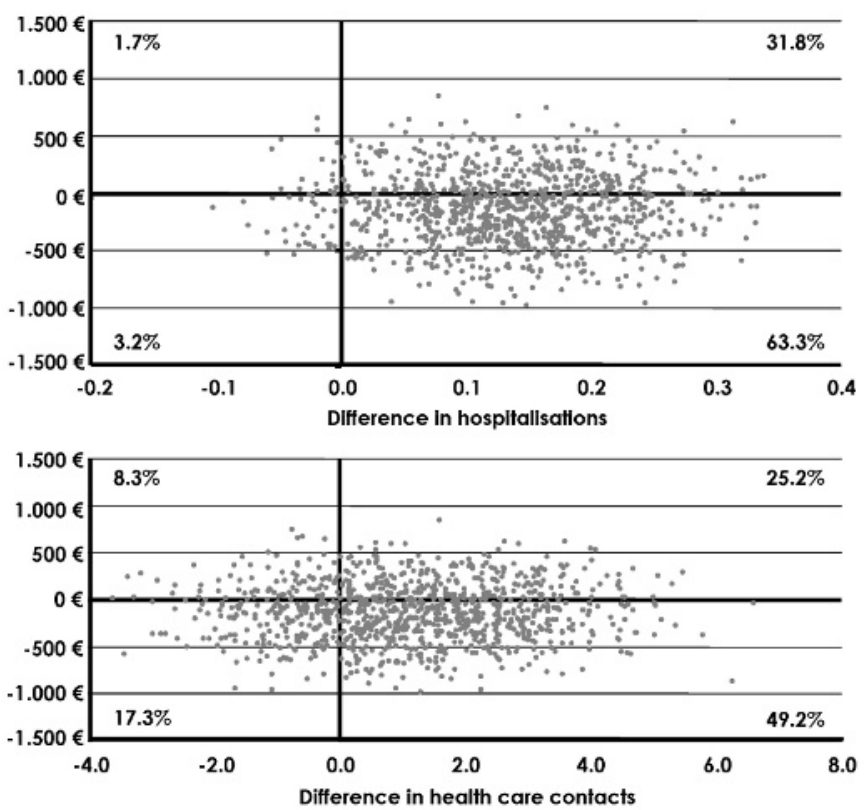

Figure 4 Results of the sensitivity analysis (1000 iterations) on cost per hospitalisation prevented and cost per health care contact prevented. A negative $€$ amount and a positive difference in the hospitalisations and health care contacts favours the self-treatment intervention. The percentages of outcomes in every quadrant are also given. instead of the change in symptoms from baseline leads to a lower number of exacerbations in both study groups. On the other hand, using symptoms from the diaries, but only as presence or absence (instead of change relative to baseline) would most likely lead to the detection of more (and more severe) exacerbations. Whether the use of these other definitions would also affect the estimates of treatment effects remains to be studied.

Using a $2 \times 2$ factorial design confers the theoretical risk of interaction between both interventions. A priori, there were no reasons to assume an interaction of a physiotherapeutic exercise programme and self-treatment of exacerbations with regard to the different outcomes. Since the proportion of patients receiving physiotherapy was the same in both study groups, possible (additional) effects of the COPE-active programme will have affected both study groups and therefore will not directly influence the between-group differences. Before the final analysis we checked for interactions between both interventions and concluded that no interaction was present.

The dominance of the self-treatment strategy was largely caused by the lower probability of hospital admission in the selftreatment group. As expected, no significant between-group difference in hospital admissions was found, since this study was not powered to detect a difference in hospital admissions.

In summary, we conclude that self-treatment incorporated in a self-management programme in patients with COPD results in fewer exacerbation days at a lower cost while not leading to overtreatment. Given the rise in the prevalence of COPD and the costs associated with treatment, we suggest that implementation programmes incorporating careful instructions on self-treatment should be started as part of self-management programmes.

Table 5 Effects per patient per year of patients with COPD receiving a self-management programme with and without self-treatment

\begin{tabular}{lccc}
\hline & $\begin{array}{l}\text { Self-treatment } \\
(\mathbf{n}=\mathbf{7 0 )}\end{array}$ & $\begin{array}{l}\text { Control } \\
(\mathbf{n}=\mathbf{7 2})\end{array}$ & Difference \\
\hline Number of exacerbation days & 42.20 & 64.50 & -22.30 \\
Number of hospitalisations & 0.200 & 0.334 & -0.134 \\
Number of health care contacts* & 5.373 & 6.513 & -1.140 \\
\hline
\end{tabular}

*Includes (telephone) consultations with the physician and general practitioner, and emergency room visits. 
Acknowledgements: We gratefully acknowledge all the members of our department of pulmonary medicine, but especially nurse practitioner Clara van Ommeren, lung function technician Irma Wissink, data managers Betty Rinsma and Petra Meerlo and, of course, the patients.

Funding: This study was funded by a grant from the Dutch Asthma Foundation. Competing interests: None.

Ethics approval: The medical ethics committee of the Medisch Spectrum Twente Hospital, Enschede, The Netherlands approved the study.

Provenance and peer review: Not commissioned; externally peer reviewed.

\section{REFERENCES}

1. Seemungal TA, Donaldson GC, Paul EA, et al. Effect of exacerbation on quality of life in patients with chronic obstructive pulmonary disease. Am J Respir Crit Care Med 1998;157(5 Pt 1):1418-22.

2. Rutten-van Molken MP, Postma MJ, Joore MA, et al. Current and future medical costs of asthma and chronic obstructive pulmonary disease in The Netherlands. Respir Med 1999;93:779-87.

3. Kessler R, Stahl E, Vogelmeier $\mathrm{C}$, et al. Patient understanding, detection, and experience of COPD exacerbations: an observational, interview-based study. Chest 2006:130:133-42.

4. Wilkinson TM, Donaldson GC, Hurst JR, et al. Early therapy improves outcomes of exacerbations of chronic obstructive pulmonary disease. Am J Respir Crit Care Med 2004:169:1298-303.

5. Anthonisen NR, Manfreda J, Warren $\mathrm{CP}$, et al. Antibiotic therapy in exacerbations of chronic obstructive pulmonary disease. Ann Intern Med 1987;106:196-204.

6. Rabe KF, Hurd S, Anzueto A, et al. Global strategy for the diagnosis, management, and prevention of chronic obstructive pulmonary disease: GOLD executive summary. Am J Respir Crit Care Med 2007;176:532-55.

7. Altman DG, Bland JM. Treatment allocation by minimisation. BMJ 2005;330:843.
8. Pieterse ME, Seydel ER, DeVries $\mathrm{H}$, et al. Effectiveness of a minimal contact smoking cessation program for Dutch general practitioners: a randomized controlled trial. Prev Med 2001;32:182-90.

9. Puhan MA, Behnke M, Laschke M, et al. Self-administration and standardisation of the chronic respiratory questionnaire: a randomised trial in three German-speaking countries. Respir Med 2004;98:342-50.

10. Juniper EF, Guyatt GH, Willan $\mathrm{A}$, et al. Determining a minimal important change in a disease-specific Quality of Life Questionnaire. J Clin Epidemiol 1994;47:81-7.

11. van der Molen T, Willemse BW, Schokker S, et al. Development, validity and responsiveness of the Clinical COPD Questionnaire. Health Qual Life Outcomes 2003; 1:13.

12. Kocks JW, Tuinenga MG, Uil SM, et al. Health status measurement in COPD: the minimal clinically important difference of the clinical COPD questionnaire. Respir Res 2006; 7:62.

13. Zigmond AS, Snaith RP. The Hospital Anxiety and Depression Scale. Acta Psychiatr Scand 1983;67:361-70.

14. Oostenbrink JB, Bouwmans CAM, Koopmanschap MA, et al. Handleiding voor kostenonderzoek. College voor zorgverzekeringen, 2004.

15. Turnock AC, Walters EH, Walters JA, et al. Action plans for chronic obstructive pulmonary disease. Cochrane Database Syst Rev 2005;(4):CD005074.

16. Monninkhof E, Van der Valk P, van der Palen J, et al. Effects of a comprehensive self-management programme in patients with chronic obstructive pulmonary disease. Eur Respir J 2003;22:815-20.

17. Langsetmo L, Platt RW, Ernst P, et al. Underreporting exacerbation of chronic obstructive pulmonary disease in a longitudinal cohort. Am J Respir Crit Care Med 2008; 177:396-401.

18. Miravitlles $\mathbf{M}$, Ferrer $\mathbf{M}$, Pont $\mathrm{A}$, et al. Effect of exacerbations on quality of life in patients with chronic obstructive pulmonary disease: a 2 year follow up study. Thorax 2004;59:387-95.

19. Spencer S, Jones PW. Time course of recovery of health status following an infective exacerbation of chronic bronchitis. Thorax 2003:58:589-93. 